Cinémas

Revue d'études cinématographiques

Journal of Film Studies

DE MOURGUES, Nicole. Le Générique de film. Paris : Méridiens

Klincksieck, 1994, 292 p.

\title{
Linda Dewolf
}

Volume 5, numéro 3, printemps 1995

URI : https://id.erudit.org/iderudit/1001155ar

DOI : https://doi.org/10.7202/1001155ar

Aller au sommaire du numéro

Éditeur(s)

Cinémas

ISSN

1181-6945 (imprimé)

1705-6500 (numérique)

Découvrir la revue

Citer ce compte rendu

Dewolf, L. (1995). Compte rendu de [DE MOURGUES, Nicole. Le Générique de film. Paris : Méridiens Klincksieck, 1994, 292 p.] Cinémas, 5(3), 187-193.

https://doi.org/10.7202/1001155ar d'utilisation que vous pouvez consulter en ligne.

https://apropos.erudit.org/fr/usagers/politique-dutilisation/ 
DE MOURGUES, Nicole. Le Générique de film. Paris: Méridiens Klincksieck, 1994, 292 p.

L'ouvrage de Nicole de Mourgues est consacré au générique de film, fragment dont les spectateurs méconnaissent trop souvent l'importance parce qu'ils sont habitués à ce que les films aient un générique et n'y prêtent plus attention: seuls les cinéphiles le regardent vraiment. Avec l'appui de nombreux exemples variés, le souci premier de ce livre consistait donc à réhabiliter le générique comme séquence filmique à part entière, voire comme séquence "majeure" ou matricielle.

Dans la première partie, l'auteure propose quelques réflexions théoriques sur le statut du générique de film, ce fragment de film situé fréquemment et de manière compacte en position inaugurale (en ouverture), parfois en position finale. Dans tous les cas, le générique est placé à la lisière, à l'orée du film, il l'enserre de sa griffe, il l'encadre, sa position frontalière fait de lui un seuil au sens de Gérard Genette (p. 3), une sorte de sas institutionnel qui facilite au spectateur le passage du monde réel au monde fictionnel et inversement. Étymologiquement, le terme générique est dérivé du latin genus-generis et signifie "genre", le générique de film mentionne les noms de ceux qui, au sein d'une équipe, ont contribué à la genèse, à la production, à la fabrication de cet objet culturel. Tout l'intérêt du générique réside justement dans cette situation complexe, paradoxale et inconfortable qui consiste à faire frontière et lien entre deux mondes: le monde réel et la fiction. Le générique présente une claire parenté avec ce que G. Genette, dans le domaine de l'écrit, nomme le paratexte; c'est le lieu où s'établit un contrat entre le film et son spectateur. Le générique est un élément du texte filmique qui, en dépit de variantes, occupe un emplacement fixe "autour du texte»: il 
comprend généralement un avant-texte et un après-texte: "[...] il est l'alpha et l'oméga du texte."

La plupart des génériques s'adressent au spectateur par écrit: ainsi, le générique est un peu l'équivalent de la notice, à ceci près que du point de vue linguistique, il est rarement un texte structuré, c'est plutôt un texte à trous, elliptique, syntaxiquement pauvre, pas vraiment narratif, mais informatif. La séquence-générique met en ouvre la réflexivité: c'est une partie du film qui s'adresse au spectateur pour lui parler du film. Avec les génériques oraux, les modalités d'adresse au spectateur sont plus variées: en voix in ou en voix off, le Mépris de J. L. Godard offrant un exemple de générique parlé célèbre dans l'histoire du cinéma.

La position inaugurale du générique dans le texte filmique permet de le comparer aux incipit de la littérature. Beaucoup d'incipit littéraires sont l'équivalent, au cinéma, des scènes d'exposition: ils situent conjointement le lieu et l'époque où vont évoluer les personnages. Ces indications précises de lieu et de temps visent à donner une impression de réalisme et de la crédibilité au récit (exemples cités: Germinal, Le Rouge et le Noir, Casablanca). La fin du générique d'Autant en emporte le vent donne des informations relatives à l'époque et au lieu de l'action. Un autre point commun entre les incipit et les débuts de films réside dans l'emploi fréquent des déictiques: Lili Marleen commence en musique par "C'est l'histoire d'une chanson ".

En tant que zone intermédiaire entre le hors-texte et le texte, le générique joue un rôle de médiateur et fonctionne pour le public comme zone d'adaptation entre le hors-texte et le texte, il fait en quelque sorte les présentations. Il est des cas où le générique est conçu comme un véritable court métrage. Dans Vertigo (Sueurs froides) d'Alfred Hitchcock, S. Bass s'est efforcé, dès le générique, de donner symboliquement au spectateur une sensation de vertige en jouant avec les couleurs, les formes et les mouvements, et à susciter l'angoisse.

Le générique, tout en appartenant au texte filmique, en constitue un fragment dont le statut est particulier: il fonctionne " comme matrice génératrice du texte" (Kuntzel, p 136). S. Bass a parlé du générique comme avant-goût symbolique de ce qui va suivre: c'est un petit film dans le film. Mais, comme les préfaces 
en littérature, les génériques sont généralement réalisés lorsque les autres fragments du film sont terminés, soit par le réalisateur lui-même (préface de type "auctoriale", cas le plus fréquent), soit par des spécialistes (allographie). La fonction première d'un générique-préface est de livrer le maximum d'informations sur l'œuvre (genèse, source, genre, récompenses obtenues...), à la situer, à guider le spectateur, à le mettre dans l'ambiance du film. Pour illustrer l'idée que le générique est non seulement une séquence filmique à part entière, mais encore parfois véritablement la matrice du film, Nicole de Mourgues cite en exemple $M$ le Maudit de Fritz Lang. Dans le texte court du générique, tous les éléments sont mis en place (images, mentions écrites, bruit, musique) que dans son déroulement ultérieur le syntagme filmique va développer, déplier, décliner.

Lieu de la nomination, le générique est aussi une forme de signature. Parmi tous les noms propres qui, au générique, évoquent ceux qui ont pris part au film, le nom du réalisateur jouit d'un prestige particulier: c'est lui qu'on considère comme l'auteur du film. Certains réalisateurs apparaissent eux-mêmes dans leurs films: dans La Mort aux trousses (1959), Hitchcock fait un pas dans la diégèse à la fin du générique.

La deuxième partie étudie comment le générique (entre)mêle ou dissocie les trois modalités du lire, du voir et de l'entendre, autrement dit, comment le générique gère les enjeux complexes du "livisaudible", ce mot valise rendant compte de l'obligation faite au spectateur de percevoir simultanément du lisible, du visible et de l'audible. Comme l'ensemble du texte filmique, cette séquence mobilise l'œil et l'oreille, mais elle a la particularité de faire grand usage de la langue.

Il convient de souligner que le générique de film est avant tout le lieu de rencontre de plusieurs systèmes sémiotiques. L'emploi d'une pomme pour la lettre o dans La Boum 2 (1982) de Claude Pinoteau montre bien que mots et images peuvent se combiner, se conjuguer allégrement à l'écran et que le texte peut aussi avoir une dimension esthétique visuelle.

$\mathrm{Ce}$ qui retiendra notre attention ici ce sont les modalités d'apparition à l'écran des inscriptions graphiques, sur l'image, dans l'image, leur forme, leur caractère esthétique plutôt que 
leur contenu. C'est la première fois que le générique de film se trouve analysé dans tous ces aspects pris un par un: taille des lettres, typographie qui caractérisent la chose imprimée, ordre des nominations, présence ou absence d'une image de fond, génériques parlés, rôle de la musique, cas particuliers célèbres comme celui de Saül Bass. Jusque dans les années trente et quarante, avant que la couleur n'envahisse les écrans, la majorité des films écrivaient leurs génériques sur un support neutre (fond gris ou brunâtre) (films de Renoir: Boudu sauvé des eaux, 1932; La Règle du jeu, 1939; La Grande Illusion, 1937). Avec le passage du noir et blanc à la couleur et du silence à la parole, ce type de cartons-génériques a bien vite été remplacé par des compositions plus sophistiquées. Les fonds sombres furent remplacés par la couleur, le dessin ( $M$ le Maudit), le fond photographique (La Mariée était en noir, 1964, de François Truffaut; Vivre sa vie, 1962, de Jean-Luc Godard), le mouvement (Les Temps Modernes, 1936, de Chaplin), les travellings (Le Signe du Lion, 1959, d'Eric Rohmer; Le Déclin de l'Empire américain, 1985, de Denys Arcand ). Dès que le cinéma s'est affranchi des cartons à fond uni et a commencé à faire preuve de fantaisie, surtout à partir des années trente, toutes les ressources de la chose écrite furent transposées dans les génériques de films (un album dans Jeux interdits, 1952, de René Clément; un programme de théâtre dans Mélo d'Alain Resnais). Si la plupart des génériques privilégient le lisible, d'autres jouent davantage avec le visible (dans James Bond contre Docteur No, 1964, de Terence Young), les mentions graphiques se superposent à un jeu de formes géométriques et de couleurs.

Dans certains films, la séquence-générique est la seule qui soit accompagnée de musique, laquelle est alors le plus souvent extradiégétique: c'est le cas dans Scarface (1932) de Howard Hawks ou dans $M$ le Maudit. La musique classique de grands compositeurs est, plus souvent qu'on ne le pense, réutilisée dans son intégrité, mais pas dans son intégralité. Certaines compositions ont marqué l'histoire de la musique de film en se faisant entendre dès le générique (Le Troisième Homme, 1954, de Carol Reed; Jeux interdits, 1952, de René Clément; West Side Story, 1961, de Robert Wise). Si la musique est l'un des ingrédients 
majeurs qui participe des éléments sonores d'un film et qui joue un rôle non négligeable pour délimiter le territoire du générique, elle n'accompagne pas seule les images, bien souvent, elle est mêlée à d'autres constituants sonores (bruits, paroles) ou entrecoupée par eux (Le Mépris; Il était une fois dans l'Ouest, 1968, de Sergio Leone). Le générique peut aussi s'accompagner d'une chanson: comme la durée de l'un coïncide facilement avec la durée de l'autre, cela donne une unité à la séquence générique.

La troisième partie aborde en détail la composition interne du générique. Autonome et en même temps dépendant du contexte filmique, le générique est analysable en plusieurs constituants: le logo et son ancêtre "la marque de fabrique", qui situent le film dans l'industrie cinématographique et donnent le coup d'envoi du film; le titre, dont les fonctions sont multiples; et le générique proprement dit, qui égrène des noms propres et des noms de métier du cinéma.

Le titre, c'est l'enseigne du film, la seule mention écrite à peu près constante du film depuis les origines du cinéma. Il a le statut d'annonceur de l'œuvre; qu'il précède toujours: il assure différentes fonctions dont celle qui consiste à désigner le film. Au cours des premières années de l'industrie cinématographique, les grandes compagnies ont créé leur style, se présentant par des logos caractéristiques qui, avec une grande diversité dans la combinatoire linguistique, iconique, voire sonore et un graphisme artistique percutant (le lion de la MGM, la marguerite de Gaumont, la montagne de Paramount), avaient en commun de borner le texte filmique (au moins une fois au début, souvent au début et à la fin).

Le titre est un "objet paratextuel " qui joue un rôle très important dans le dispositif de prise de contact entre le film et son spectateur, dit Nicole de Mourgues. Le titre relève du code de la langue. En outre, il a un statut intéressant parce que double: il fait corps avec l'œuvre qu'il étiquette, il est véritablement le nom propre du film, et tout en étant intimement lié à l'œuvre, le titre mène une vie autonome, il représente le film à l'extérieur. Ce double statut permet de lui attribuer plusieurs fonctions: une fonction publicitaire, une fonction référentielle 
(le titre fonctionne comme une synecdoque), une fonction désignative et souvent une fonction descriptive. Certains titres ont une fonction pragmatique ou conative et d'autres travaillent les fonctions poétique et esthétique.

Sous une grande variété de constructions syntaxiques, la spécificité du titre de film réside essentiellement dans sa nécessaire concision, son caractère elliptique et dans l'emploi massif de syntagmes nominaux allant des simples nom ou prénom (Carmen, Loulou, Lola, Ninotchka, Viridiana, Bambi, Mickey, Jules et Jim, Camille Claudeh Barton Fink, Thèrese Raquin); surnoms: Zaza, Lili; noms propres de pays: Allemagne, année zéro, Casablanca, Paris-Texas...; à des groupes syntaxiques: Madame du Barry, Le Baron de Münchhausen, Les Saeurs Brontë. Beaucoup de films identifient une entité, par exemple La Lectrice, Le Trou, Les Oiseaux, Cette sacrée gamine, Mon père, Trois Femmes, avec une grande fréquence de titres binaires qui associent deux syntagmes nominaux: Le Festin de Babette. Même si les syntagmes nominaux sont de loin les plus usités comme titres de films, il n'en reste pas moins que certains titres sont des phrases indépendantes assertives, interrogatives, exclamatives, impératives... les phrases ancrent le titre (et le film à venir) dans un contexte spatio-temporel précis: J'accuse, Le jour se lève, Et Dieu créa la femme, Qui a peur de Virginia Woolf?, Occupe-toi d'Amélie!, Que viva Mexico!

Qu'il soit bref, concis et elliptique, on attend du titre qu'il livre des informations, aussi minimales soient-elles, sur le contenu du film. Qu'on le veuille ou non, le titre de film donne une piste de lecture, c'est "une clé interprétative». Étant donné l'importance de l'impact du titre sur le spectateur, les réalisateurs ont souvent recours à des figures de rhétorique telles que l'allitération, la métaphore (Le Guépard), le palindrome, la synecdoque pour que leurs titres soient remarqués et mémorisés. Enfin, le titre de film a un statut particulier dans la mesure où c'est lui qui, assorti ou non au nom du réalisateur, représente le film à l'extérieur; on s'efforce en général de le mettre en valeur, essentiellement par le choix de son emplacement et de son graphisme. Statistiquement, le titre est situé le plus souvent en amont du film, il tient ainsi son rôle de "chapeau", d'enseigne 
du film. Il suit presque toujours le logo ou d'autres informations concernant la production. Mais l'emplacement du titre est sensible à des phénomènes de mode, aussi la mention du titre après les noms des acteurs et du réalisateur est moins fréquente. Quelle que soit sa forme linguistique, le titre est la partie la plus diégétique, voire diégétisée du générique.

Le générique proprement dit est le fragment filmique qui, de manière virtuelle et dans un style en général elliptique, apporte des informations sur le texte filmique qu'il encadre. De nos jours, le générique type est scindé en deux parties: le générique de début et le générique de fin qui présentent tous deux les noms propres et les noms de métier de tous ceux qui ont fait le film; c'est le lieu de la nomination des gens du film: les stars, le réalisateur, les autres acteurs, le producteur, le scénariste, le musicien et ou compositeur et les autres techniciens du son, le décorateur, le monteur, les autres collaborateurs et enfin les prix, les récompenses et les dédicaces. Au terme de tout ce processus de "génération", il apparaît clairement que le générique, c'est - déjà ou encore - le film.

Linda Dewolf

Université de Liège

\section{OUVRAGES CITÉS}

Genette, Gérard. "Présentation ". "Paratextes ", Poétique, n 69.

Kuntzel, Thierry. "Le travail du film". "Le texte, de la théorie à la recherche", Communications, $\mathrm{n}^{\circ} 19$ (1975), p. 25-39. 